Pädiatrie und Pädologie 2013 · 48:5

DOI 10.1007/s00608-013-0062-x

C) Springer-Verlag Wien 2013

\author{
B. Resch \\ Univ. Klinik für Kinder- und Jugendheilkunde, Medizinische Universität Graz
}

\title{
13. Pädiatrischer Frühling
}

Es freut mich, dieses Editorial im seit 2013 neuen Design von Pädiatrie \& Pädologie zu verfassen und mit dieser Ausgabe den 13. Pädiatrischen Frühling einläuten zu dürfen. Für Prof. Müller und mich bedeutet diese seit Jahren ausgebuchte Fortbildungsveranstaltung, die zu den drei großen österreichischen Pädiatrietagungen zählt, eine immer wieder neue Herausforderung und Freude in der Organisation und Durchführung. Mit seiner Lage inmitten einer alten historischen Kulturlandschaft im südsteirischen Weinland und durch seine Abgeschlossenheit bietet der Veranstaltungsort Schloss Seggau eine ideale Fortbildungsstätte. In diesem herrlichen Ambiente wird den niedergelassenen wie auch im Spital tätigen Fachärzten für Kinder- und Jugendheilkunde und solchen in Ausbildung eine intensive Wissensauffrischung und Kommunikation ermöglicht. Wir laden somit wiederholt zu einer Auffrischung persönlicher Kontakte und zum Gedankenaustausch unter Kolleginnen und Kollegen herzlich ein. Diesem kollegialen Motto wird in den zweieinhalb Tagen Fortbildung auch lebhaft nachgekommen. Bereits traditionell gibt es eine große Industriebeteiligung mit zahlreichen Ausstellern, die in den Pausen bei Getränken und Brötchen bzw. Kaffee und Kuchen zwanglos mit der Kollegenschaft kommunizieren können.

Das Konzept des Pädiatrischen Frühlings beinhaltet kinderärztliche Fortbildung mit einerseits Seminaren und Workshops und somit Arbeit in Kleingruppen und andererseits Plenarsitzungen mit Vorträgen, die auch ausgiebig Raum für Diskussion lassen. Um dieser Diskussion nachkommen zu können, ha- ben wir die Zahl der Vortragenden etwas reduziert, um noch fokussierter bei etwas weniger Themen verweilen zu können. Ein großes Anliegen ist uns, unmittelbar von der Veranstaltung die sogenannten „take-home messages“ mitnehmen zu können und die Wissensvermittlung sehr praxisrelevant aufzubauen. Dies wird uns auch von den Teilnehmern in der immer sehr kritischen Evaluierung positiv rückgemeldet. Zusätzlich zum Programm in Seggau wird ein eintägiger Sonographieauffrischungskurs an unserem „Grazer Kinderzentrum“ unter der bewährten Leitung von Univ.-Prof. Dr. M. Riccabona (ÖGUM/DEGUM Ausbilder von der Klinischen Abteilung für Kinderradiologie Graz) angeboten, der die positive Kooperation von Kinderklinik, Kinderchirurgie und Kinderradiologie unter Beweis stellt. Die Tagung beginnt immer Donnerstag früh und endet am Samstag zu Mittag mit einer Abschiedsjause. Workshops und Seminare werden vierstündig oder auch ganztägig abgehalten und finden am ersten Kongresstag statt, sodass die Teilnehmer bis zu zwei Kurse an diesem Tag besuchen können. Die Möglichkeit der Teilnahme am ganztägigen Sonografiekurs wird durch ein Shuttleservice von Seggau nach Graz und retour unterstützt.

Unsere Workshopreihe besteht aus einem bunten Angebot an wiederholten Kursen und immer wieder neuen Themen. Heuer haben wir im Rahmen der Entwicklungspädiatrie den gesamten Komplex der Frühgeborenennachsorge eingebaut und bringen fächerübergreifende Workshops wie die Kinderdermatologie und Kinderorthopädie. Weiters bieten wir neue Workshops in Kindernephro- logie und pädiatrischer Infektiologie mit Schwerpunkt Antibiotikatherapie.

In den letzten Jahren hat sich am Freitag ein Lunchsymposium etabliert, das trotz der intensiven Fortbildungszeit von den Kongressteilnehmern sehr positiv angenommen worden ist.

Mit den Plenarthemen hoffen wir den Teilnehmern ein breites Spektrum aus der Pädiatrie ausgewählt zu haben. Der Bogen reicht vom Zusammenhang von Schlaf, Schlafstörungen und Schulleistung über das obstruktive Schlafapnoesyndrom, die Fremdkörperaspiration (Variabilität in der klinischen Präsentation und Pitfalls in Diagnostik und Therapie), die Diagnose und Therapie der Bienen- und Wespengiftallergie, endokrine Notfälle, prämature Thelarche, Gynäkomastie, Pubertas präcox, Sinusitis, Orbitalphlegmone und deren Komplikationen (Sichtweisen vom pädiatrischen Infektiologen und HNO-Spezialisten), die Hantavirus-Infektionen (vorgestellt vom pädiatrischen Nephrologen) bis zur pädiatrischen Zahnheilkunde mit Karies und all ihren Facetten und den Zahn- und Kieferfehlstellungen. Den Abschluss der Tagung bieten die Fallpräsentationen von Prof. Kerbl aus Leoben und OA. Sauseng von Graz mit der Aufforderung an alle Kongressteilnehmer: „Stellen Sie die Diagnose!“

Unter dem Motto „non recuso laborem“ möge der gute Fortbildungsgeist von Schloss Seggau bei unserer Veranstaltung wie auch beim Blättern und Lesen dieser Ausgabe von Pädiatrie \& Pädologie wirken.

Ihr Bernhard Resch

Email: bernhard.resch@medunigraz.at 